\title{
External iliac vein injury during total hip arthroplasty: a case report
}

\author{
Ji Hyo Kim*, Ji Hyun Kim*, Hyeon Jun Lee, Yehun Jin, Seong Wook Hong \\ Department of Anesthesiology and Pain Medicine, Kyungpook National University Hospital, Daegu, Korea
}

Received August 1, 2018

Revised August 9, 2018

Accepted August 10, 2018

Corresponding author

Seong Wook Hong

Department of Anesthesiology and

Pain Medicine, Kyungpook National

University Hospital, 130 Dongdeok-

ro, Jung-gu, Daegu 41944, Korea

Tel: $+82-53-420-5872$

Fax: +82-53-426-2760

E-mail: hsuaa@naver.com

ORCID:

http://orcid.org/0000-0002-4849-4738

*Ji Hyo Kim and Ji Hyun Kim

contributed equally to this work and

also co-first authors.

\begin{abstract}
Vascular injuries during total hip arthroplasty (THA) are rare, but they could be life threatening when they occurs. Therefore, early diagnosis and appropriate management should be required to reduce the rate of morbidity and mortality of the patient. In case of external iliac vein (EIV) injury, it could be more difficult to be noticed because of its slow onset, which could result in catastrophic, delayed hemorrhagic shock. Although EIV injury during THA is uncommon and has not been emphasized enough in anesthetic field, subsequence could be fatal. Herein, we report a case of EIV injury occurred by a drill tip passing through the acetabulum during THA. The patient showed an abrupt hemodynamic collapse without visual evidence of massive bleeding. Cardiopulmonary resuscitation was even required before detection and repair of EIV.
\end{abstract}

Keywords: Iliac vein injury; Total hip replacement; Hypovolemia; Shock

\section{INTRODUCTION}

Vascular complications associated with total hip arthroplasty (THA) has been reported to have a frequency of $0.1 \%$ to $0.3 \%[1,2]$. However, they could result in limb loss or life threatening condition when they occurs [3-5]. THA includes several procedures, such as drilling, reaming, cementing, and the fixation of screws that penetrate the inner cortex of the pelvis and may damage pelvic vascular structures [6,7]. In particular, the external iliac, femoral, and obturator vessels are at the greatest risk of injury because of their proximity to the acetabulum. We report a rare case of external iliac vein (EIV) injury during THA under epidural anesthesia.

\section{CASE REPORT}

A $153 \mathrm{~cm}, 53 \mathrm{~kg}$, 64-year-old woman with chronic osteoarthritis was admitted for left THA under epidural anesthesia. She was a hepatitis B virus carrier, but otherwise healthy. Her preoperative examination was unremarkable. In the operating room, standard monitoring devices were applied. Initial vital signs of the patient were stable, showing blood pressure (BP), heart rate (HR) and oxygen saturation $\left(\mathrm{SpO}_{2}\right)$ of $134 / 69 \mathrm{mmHg}, 87 \mathrm{bpm}, 100 \%$, respectively. Her 
electrocardiography (ECG) showed normal sinus rhythm. Lactated Ringer's solution was administered via 20-guage peripheral line placed from ward. Lumbar epidural analgesia was planned for the operation. An 18-gauge Tuohy epidural needle (Perifix ${ }^{\circledR}$; B.Braun Melsungen AG, Melsungen, Germany) was inserted into the lumbar epidural space at the L4-5 level in left lateral decubitus position. Using the loss of resistance (LOR) technique, the epidural space was identified at a depth of $5 \mathrm{~cm}$ from the skin on the first attempt. After ensuring that there was no cerebrospinal fluid (CSF) or blood leak, an epidural catheter (Perifix ${ }^{\circledR}$ ) was inserted and we slowly injected $90 \mathrm{mg}$ of $0.75 \%$ ropivacaine. After 15 minutes, the level of analgesia reached the 10th thoracic dermatome of the patient. She was positioned in the right lateral decubitus position for the surgery and sedated with $2.5-3 \mathrm{mg} / \mathrm{kg} / \mathrm{h}$ of $2 \%$ propofol intravenously. After positioning, the patient's vital signs were as follows: $\mathrm{BP}$, $\mathrm{HR}$ and $\mathrm{SpO}_{2}$ of 101/58 mmHg, $67 \mathrm{bpm}$ and 100\% under face oxygen mask with a flow of $5 \mathrm{~L} / \mathrm{min}$, respectively. The patient's vital signs remained stable at 25 minutes after skin incision when the drilling started. 40 minutes after skin incision, the patient's BP decreased slightly from 111/61 mmHg to $82 / 53 \mathrm{mmHg}$ with $\mathrm{HR}$ of $70 \mathrm{bpm}$. After injection of 0.1 mg of phenylephrine and starting the administration of 500 mL of hydroxyethyl starch (Volulyte ${ }^{\circledR}$ 6\%; Fresenius Kabi Deutschland GmbH, Bad Homburg, Germany), the patient's $\mathrm{BP}$ increased to $118 / 71 \mathrm{mmHg}$.

However, 70 minutes after, her BP decreased abruptly to $68 / 34 \mathrm{mmHg}$ with HR of $78 \mathrm{bpm}$. As we continued fluid resuscitation, $0.1 \mathrm{mg}$ of phenylephrine was injected immediately twice, but her BP remained as low as $87 / 52 \mathrm{mmHg}$ and HR started to increase from 80 to $100 \mathrm{bpm}$ (Fig. 1). The propofol infusion for sedation was stopped and continuous phenylephrine infusion was started at a rate of $0.5 \mu \mathrm{g} /$ $\mathrm{kg} / \mathrm{min}$. We immediately placed a 20 -guage catheter in the left brachial vein for additional intravenous access and administered $500 \mathrm{~mL}$ of hydroxyethyl starch (Volulyte ${ }^{\circledR} \%$ ) again. Despite our efforts, her BP did not increase properly and the patient began to complain of chest discomfort and dizziness at this point. The blood volume contained in suction bottles, wet gauzes showed the amount of only $370 \mathrm{~mL}$ approximately and there had been little bleeding observed in the operating field during the surgery. However, her BP dropped to $45 / 27 \mathrm{mmHg}$ eventually and her conjunctivae looked pale. We could suspect hypovolemic shock, pulmonary thromboembolism, or myocardial infarction in the presence of profound hypotension and drowsy mental status of the patient.

The surgeons agreed to stop the surgery and moved the patient into the supine position for further investigation of the cause and management. Right after the supine position, the patient's abdomen was inflated and her BP suddenly became undetectable with ECG showing bradycardia (HR of 43 bpm) with normal sinus rhythm (Fig. 1). $100 \mu \mathrm{g}$ of epinephrine was administered, but BP did not recover and pulsation of the patient became significantly faint showing pulseless electrical activity. Therefore, cardiopulmonary resuscitation (CPR) was performed immediately in a Trendelenburg position with external cardiac compression, manual ventilation with 100\% oxygen, and rapid hydration. Epinephrine $1 \mathrm{mg}$ was injected for 3 times every 5 minutes repeatedly. As continuing CPR, we performed endotracheal intubation and right femoral artery was cannulated and connected to a Vigileo ${ }^{\mathrm{TM}}$ monitor with FloTrac sensor (Edwards Lifesciences LLC; One Edwards Way, Irvine, CA, USA) for estimating cardiac index, stroke volume variation as well as BP continuously. Arterial blood gas analysis failed to check hemoglobin level at this point showing watery color than it normally should be. Central venous catheterization was also performed immediately with a MAC ${ }^{\mathrm{TM}}$ (Multi-lumen Access Catheter, 9-French; ARROW International Inc., Reading, PA, USA) and three lumen central venous catheter (Blue FlexTip ${ }^{\circledR}$ ARROWg+ard Blue ${ }^{\circledR}$ Catheter, 7-French; ARROW International, Inc., Reading, PA, USA) in the right internal jugular vein and we started volume resuscitation with a rate of 50 $\mathrm{mL} / \mathrm{min}$ using Belmont FMS 2000 Rapid Infuser (Fluid Management System; Belmont Instruments, Billerica, MA, USA). Continuous infusion of norepinephrine $(0.2 \mu \mathrm{g} / \mathrm{kg} / \mathrm{min})$ and vasopressin ( $0.1 \mathrm{unit} / \mathrm{min}$ ) were applied. Vascular surgeons were consulted to examine the patient's abdomen, and copious bleeding was detected in the retroperitoneal area. The left EIV and some smaller veins of the patient had been found to be transected. Because the left EIV was crushed, the vascular surgeons could not perform an excision with end-to-end anastomosis, and they decided to tie off the proximal and distal stumps. CPR continued for 30 minutes until vascular surgeons clamped the injured vessel while we performed volume resuscitation with vasopressor support. 


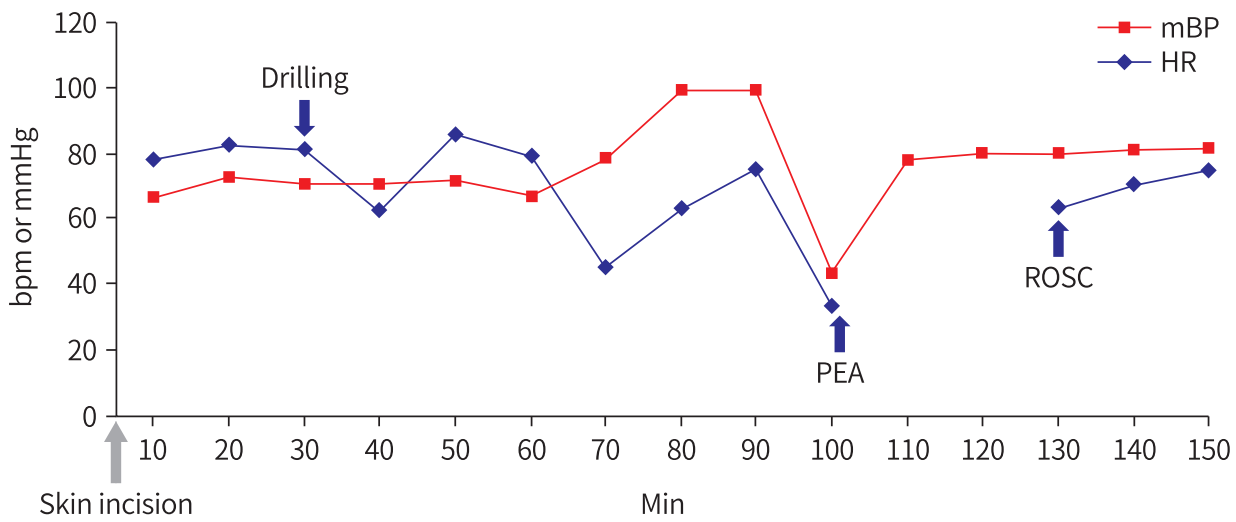

Fig. 1. Intraoperative change in mean blood pressure and heart rate. PEA: pulseless electrical activity, ROSC: return of spontaneous circulation, mBP: mean blood pressure, HR: heart rate.
After return of spontaneous circulation (ROSC), the patient's BP recovered to $81 / 54 \mathrm{mmHg}$ with a HR of 80 (Fig. 1). Hemodynamic values from FloTrac sensor were hardly detected during CPR, however, showed cardiac index (CI) and stroke volume variation of $2.0 \mathrm{~L} / \mathrm{min} / \mathrm{m}^{2}$ and $18 \%$ respectively after ROSC.

Patient received 23 units of packed RBCs, 12 units of fresh frozen plasma, and 20 units of platelets, $5 \mathrm{~L}$ of crystalloid solution, and $3 \mathrm{~L}$ of colloid solution totally during 7 hours of this operation. The estimated total blood loss was $16 \mathrm{~L}$, and the total urine output was $6.6 \mathrm{~L}$.

At the end of the surgery, her vital signs were stabilized and she was transferred to the surgical intensive care unit. The results of the postoperative laboratory result showed hemoglobin of $9.2 \mathrm{~g} / \mathrm{dL}$, hematocrit of $27.6 \%$, platelets of $230,000 / \mu \mathrm{L}$, prothrombin time of 14.4 seconds and international normalized ratio of 1.33 . Her mental status was restored in 2 hours, and she was extubated 12 hours after surgery. On the fifth postoperative day, the results of follow-up ECG, myocardial enzyme estimation, and blood tests were normal and the patient showed no symptoms or complications. The patient was transferred to a general ward after recovery and discharged 2 months after because her abdominal wound healing was delayed. The patient's consent was obtained for publication of this case report.

\section{DISCUSSION}

Although THA is considered as a safe surgical procedure, serious and even fatal complications could occur [8]. Vascular injuries in THA are rare, however, complication from the event could compromise the outcome of operation [9] showing catastrophic hemodynamic alterations [3-5]. THA necessitates the placement of retractors around the acetabulum where extensive vasculature passes. Therefore direct vessel injury during THA could occur as a result of placement of supplemental screws for fixation of the acetabular component [6,10]. In our case, the patient's left EIV was transected as a result of predrilling holes for the anchoring of screws and pegs. External iliac vein is one of the most commonly injured vessels during THA with external iliac artery (EIA) and common femoral artery [1]. Between external iliac artery and vein, because of more medial position and less interposed tissue along the pelvic brim which could protect vessels, EIV seems more vulnerable than EIA [7]. However, injuries related to EIV has been reported much less than EIA [1,8]. The reason for this phenomenon could be explained by comparing the nature of artery and vein. Although arterial injuries tend to show dramatic alterations of vital signs and faster blood pooling in operation field, which could be easier to be detected, venous injuries usually go unnoticed, regardless of the size of the laceration, and can lead to slow onset of shock $[4,5,11]$. Besides, as Doi et al. reported [4], in lateral decubitus position, lower blood pressure may not be present or could be concealed than it actually is, which make it even more difficult to detect the presence of EIV injury during THA.

In our case, the patient showed abrupt hemodynamic collapse 70 minutes after skin incision. It was challenging for us to predict that delayed shock would occur. We could suspect hypovolemia, myocardial infarction, pulmonary thromboembolism or severe hypotension developed by neural axial block as the cause of this event, at first. Consider that ECG recording showed sinus rhythm without any isch- 
emic change, and deep vein thrombosis was not identified on the preoperative computed tomography venogram, both myocardial infarction and pulmonary thromboembolism appeared to be less likely for the cause. Profound hypotension caused by neural axial block could be also eliminated because we confirmed the level of analgesia reached before starting the surgery. Additionally, time of hemodynamic collapse occurred much later than the onset time of epidural analgesia. Even though excessive bleeding did not observed visually, in the suction bottle or surgical field, hypovolemic shock was highly suspicious based on vital sign changes including decreased BP, increased HR, and decreased urine output. It became more severe after changing the posture into supine position with patient showing inflated abdomen and pale conjunctivae. After starting CPR and general anesthesia was induced, we applied transesophageal echocardiogram (TEE) for further investigation. We could observe severe hypovolemic status with normal myocardial contractility on TEE. There was no sign of right ventricular dilatation, dysfunction or pulmonary thromboembolism. For identification and management of the situation, vascular surgeons cooperated with orthopedic surgeons and EIV injury was detected after retroperitoneal inspection.

Because damage to veins developed slow and gradual bleeding, vital signs seemed to be stable before the abrupt hemodynamic alteration. A slight decrease in BP and increase in HR were checked after beginning of the operation, and we understand that those might be the signs for decreased circulation volume. But, in usual, the neural axial block and the sedative effect of propofol are also related to that pattern of vital sign change. And because there was not much excessive bleeding seen in the field or bottle, the delayed shock by vein injury was hardly detectable or predictable before it happens. Additionally, retroperitoneal hemorrhage which is occurred by various causes is often nonspecific, therefore diagnosis is delayed, and missed sometimes [12,13]. CT and arteriography are known to be important technique for diagnosis, but those are not available immediately during operation like our case. However, we could suspect that there would be some bleeding spots based on decreased hemoglobin and hemodynamic instability even though massive bleeding was not detected clearly in the operative field. As immediate retroperitoneal inspection seemed to be required, vascular surgeons were consulted for it. Those features of vein injury and retroperitoneal hemorrhage became the blind spots along with lateral decubitus position, and the consequence was devastating. Despite a sufficient volume supply and the use of a vasopressor, it was difficult to restore the patient's vital sign and even CPR was required in our case.

THA has been grown in orthopedic field as a result of widened indications and increased life expectancy [5]. Therefore, it is important for anesthesiologists to pay more attention on the complications related to it. EIV injury in THA is uncommon event even for vascular surgeons [4]. But, we should aware that it is life threatening and could be hard to be noticed especially with lateral decubitus position [4,5,11]. However, despite the fatal risks, it still has not been emphasized enough in anesthetic literature. Herein, we report this case of EIV injury during TAH resulted in delayed hemorrhagic shock, that it might help early diagnosis and immediate management for similar events. It also needs to be considered that many of the patients who undergo THA are usually in older age group and related to various underlying disease, which make them more vulnerable to hemodynamic changes. Therefore, anesthesiologists should be vigilant and prepared for immediate identification and appropriate management in case of severe hypotension during THA to reduce morbidity and mortality of patients, and also aware of the possibility of EIV injury which could result in delayed shock, although its frequency is rare.

\section{CONFLICT OF INTEREST}

No potential conflict of interest relevant to this article was reported.

\section{REFERENCES}

1. Shoenfeld NA, Stuchin SA, Pearl R, Haveson S. The management of vascular injuries associated with total hip arthroplasty. J Vasc Surg 1990;11:549-55.

2. Müdüroğlu A, Kayhan TO, Yüksel A. Iliac vein injury during total hip replacement: a rare iatrogenic complication and its successful surgical treatment. Int J Vasc Surg Med 2017;3:335.

3. Bergqvist D, Carlsson AS, Ericsson BF. Vascular complications after total hip arthroplasty. Acta Orthop Scand 1983;54:15763. 
4. Doi S, Motoyama $\mathrm{Y}$, Itoh $\mathrm{H}$. External iliac vein injury during total hip arthroplasty resulting in delayed shock. Br J Anaesth 2005;94:866.

5. Lee KW, Jo YH, Yi JW. Threatening external iliac vein injury during total hip arthroplasty. J Kyung Hee Univ Med Cent 2011;27:60-4.

6. Keating EM, Ritter MA, Faris PM. Structures at risk from medially placed acetabular screws. J Bone Joint Surg Am 1990;72:509-11.

7. Wasielewski RC, Galat DD, Sheridan KC, Rubash HE. Acetabular anatomy and transacetabular screw fixation at the high hip center. Clin Orthop Relat Res 2005;438:171-6.

8. Parvizi J, Mui A, Purtill JJ, Sharkey PF, Hozack WJ, Rothman RH. Total joint arthroplasty: When do fatal or near-fatal complications occur? J Bone Joint Surg Am 2007;89:27-32.

9. Aust JC, Bredenberg CE, Murray DG. Mechanisms of arterial injuries associated with total hip replacement. Arch Surg
1981;116:345-9.

10. Wasielewski RC, Cooperstein LA, Kruger MP, Rubash HE. Acetabular anatomy and the transacetabular fixation of screws in total hip arthroplasty. J Bone Joint Surg Am 1990;72:5018.

11. Hwang SK. Vascular injury during total hip arthroplasty: the anatomy of the acetabulum. Int Orthop 1994;18:29-31.

12. Wang Y, Chang H, Wang W, Wang W, Liu B, Li Z, et al. Delayed retroperitoneal arterial hemorrhage after inferior vena cava filter deployment: a case report. Medicine (Baltimore) 2018;97:e9618.

13. Rana MA, Mady AF, Jakaraddi N, Mumtaz SA, Ahmad H, Naser K. Not all acute abdomen cases in early pregnancy are ectopic; expect the unexpected: renal angiomyolipoma causing massive retroperitoneal haemorrhage. Case Rep Crit Care 2016;2016:5643470. 